\title{
Aide au suivi du phénomène de dénitrification naturelle pour une gestion durable de la ressource en eau du Béthunois
}

José Luis Martin-Bordes, Éric Henry, Cherif Boulemia et François-Xavier Masson

\section{(2) OpenEdition}

Édition électronique

URL : http://journals.openedition.org/developpementdurable/2791

DOI : 10.4000/developpementdurable.2791

ISSN : 1772-9971

Éditeur

Association DD\&T

Référence électronique

José Luis Martin-Bordes, Éric Henry, Cherif Boulemia et François-Xavier Masson, « Aide au suivi du phénomène de dénitrification naturelle pour une gestion durable de la ressource en eau du Béthunois », Développement durable et territoires [En ligne], Dossier 6 | 2006, mis en ligne le 01 novembre 2006, consulté le 19 avril 2019. URL : http://journals.openedition.org/ developpementdurable/2791; DOI : 10.4000/developpementdurable.2791

Ce document a été généré automatiquement le 19 avril 2019.

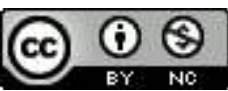

Développement Durable et Territoires est mis à disposition selon les termes de la licence Creative Commons Attribution - Pas d'Utilisation Commerciale 4.0 International. 


\title{
Aide au suivi du phénomène de dénitrification naturelle pour une gestion durable de la ressource en eau du Béthunois
}

\author{
José Luis Martin-Bordes, Eric Henry, Cherif Boulemia et François-Xavier \\ Masson
}

1 Les collectivités locales sont confrontées à des problèmes de gestion de la ressource en eau souterraine liés à la pollution anthropique et dans certains secteurs à la surexploitation. En effet, des actions locales de gestion raisonnée et de préservation sont aujourd'hui de plus en plus nécessaires pour pallier les effets négatifs d'une augmentation de population et d'activité industrielle. Néanmoins, les outils de gestion et d'aide à la décision sont rarement utilisés chez les gestionnaires de la ressource, notamment dans les moyennes collectivités où les compétences, les outils et les moyens humains et financiers sont limités (Boulémia et al., 2000). La compréhension des phénomènes naturels qui ont lieu dans les aquifères exploités pour l'alimentation en eau potable est possible aujourd'hui grâce aux outils de modélisation et de simulation, mais certains développements sont nécessaires pour adapter ces outils aux stratégies de prospective et d'aide à la décision dans une perspective d'exploitation durable de la ressource (Martin et al., 2002). Nos travaux d'investigation s'inscrivent dans un projet de partenariat avec le SIVOM (Syndicat Intercommunal à Vocation Multiple) de la Communauté du Béthunois ${ }^{1}$.

2 L'approche traditionnelle de la gestion des ressources en eau a mis l'accent sur la satisfaction des besoins en eau de l'homme, aussi bien sur le plan de la quantité que sur le plan de la qualité de l'eau. La crise de l'eau et les problèmes liés à l'environnement ont conduit à un changement radical dans la gestion de cette ressource, évoluant d'une approche technico-économique traditionnelle vers une approche de gestion durable des ressources en eau (Ganoulis, 2001). On peut discerner cinq facteurs principaux qui conduisent ensemble à la métamorphose du panorama de la gestion des ressources en eau 
(en particulier celles en eau souterraine) et à la modification des pratiques d'exploitation connues jusqu'à présent. Ces facteurs sont :

1. le changement climatique et les périodes de sécheresse,

2. le développement des villes, l'augmentation de la population et des activités humaines,

3. l'exploitation intensive des ressources en eau souterraine,

4. la dégradation de la qualité de l'eau,

5. le partage des ressources et les conflits sur l'eau.

Dans une optique de développement durable des territoires, l'identification de critères environnementaux et leur intégration dans tout processus de prise de décision sont des étapes essentielles pour garantir la préservation de la ressource face aux contraintes générées par les facteurs évoqués ci-dessus. Bien évidemment, les enjeux du territoire au niveau local ne sont pas les mêmes qu'aux niveaux régionaux, nationaux et globaux, et les échelles d'étude menant à une prise de décision et à la définition de stratégies de gestion durables doivent être adaptées à la dimension du territoire et aux spécificités locales.

4 Par la désignation de "collectivité de taille moyenne", nous entendons les collectivités dont la population se situe entre 20000 et 200000 habitants (d'après Pornon, 1998). Elles se caractérisent, en général, par :

1. une structure peu informatisée ou en voie d'informatisation,

2. une dispersion de l'information et peu de capitalisation de cette dernière (Boulémia et al., 2000).

5 L'augmentation des performances et la baisse des coûts des technologies informatiques leur autorisent désormais l'accès et l'utilisation d'outils et de technologies adaptés pour faciliter non seulement la résolution de problèmes de gestion quotidienne de la ressource en eau, mais aussi la planification à moyen et long termes (Top et al., 2000). Les enjeux sont d'une part, d'acquérir l'autonomie suffisante pour développer leurs propres stratégies d'exploitation et d'aide à la décision et d'autre part, de capitaliser et d'analyser l'ensemble des données nécessaires à l'étude de la ressource.

6 Le SIVOM de la Communauté du Béthunois gère 32 compétences techniques dont la production et la distribution d'eau potable dans 13 communes sur une superficie de 51 $\mathrm{km}^{2}$. La population desservie en 2004 était de 40.000 habitants. La collectivité, dans un souhait de préservation de la ressource, tout en garantissant les demandes en eau, a besoin d'outils appropriés pour comprendre le fonctionnement de la nappe, pour modéliser et suivre sa réponse face à une éventuelle exploitation de nouveaux ouvrages hydrauliques. En effet, une surexploitation de l'aquifère calcaire pourrait avoir un impact sur les phénomènes naturels ayant lieu dans l'aquifère, en particulier le phénomène de dénitrification naturelle ( $\$$.) qui est un phénomène d'intérêt régional et qui mérite d'être préservé. Un abaissement trop important du niveau de la nappe suite à des pompages intensifs pourrait générer d'autres effets environnementaux négatifs sur le territoire du Béthunois tels que l'assèchement des zones humides et la disparition des écosystèmes associés, et encore l'affaissement des terrains avec des dégâts sur les habitations avec des conséquences socio-économiques considérables.

7 Le Béthunois est un territoire qui a des atouts au niveau de sa ressource en eau souterraine, un aquifère productif, bien protégé des pollutions de surface et qui bénéficie d'un processus de dénitrification naturelle, cité ci-dessus. Le secteur du Béthunois dispose d'une source d'eau potable qui s'avère de bonne qualité et qui intéresse les communautés 
de communes voisines moins bien desservies. Des outils de connaissance du territoire, tant en surface qu'en profondeur, sont donc essentiels pour parvenir à une politique de gestion durable de la région dans un esprit de partage de la ressource mais également dans une optique de protection et de développement.

Notre objectif est de proposer une démarche de gestion de la ressource en eau souterraine qui doit répondre à des besoins à court, moyen et long termes pour une meilleure exploitation de la ressource. Nos sous-objectifs sont :

1. d'avoir une meilleure connaissance de la ressource en général et du fonctionnement des phénomènes naturels en particulier ;

2. d'anticiper le comportement de la ressource vis-à-vis des contraintes externes ;

3. de proposer une exploitation et une surveillance raisonnée de la ressource et de faciliter l'activité du gestionnaire d'aquifère souhaitant sa préservation.

L'orientation envisagée pour répondre à cet objectif est basée sur l'utilisation d'outils informatiques spécifiques et repose sur l'expérimentation de ceux-ci sur une application concrète (\$ 4). La plate-forme logicielle (cf. Fig.1) qui instrumente notre approche est composée de :

1. un SIG-logiciel, noyau de la plate-forme qui, grâce à ses fonctionnalités, permet non seulement la visualisation et l'agrégation des données invariables (profils géologiques, localisation et profondeur des forages d'eau, ...), mais aussi l'analyse de variables fonction du temps et de l'espace (pluviométrie, niveaux d'eau, chimie de l'eau,...) (Villeneuve et al., 1995) (Crausaz et Musy, 2001) (Waaub, 2001),

2. un tableau de bord, interfacé avec le SIG et dont la fonction principale est de fournir au gestionnaire une vision de l'ensemble des indicateurs du système hydrologique (ex. teneur en nitrate des eaux) et l'indice de priorité de l'indicateur (ex. "normal», "critique», « alerte »),

3. un logiciel de modélisation du système hydrologique, qui permet d'interpréter le fonctionnement de l'aquifère et de simuler différents scénarios hydrodynamiques et de transport de polluants, (ex. GMS ${ }^{\oplus}$, FEFLOW ${ }^{\oplus}, .$. ),

4. les bases de données physiques et dynamiques qui alimentent les modules évoqués ci-dessus.

Figure 1. Plate-forme logicielle instrumentant l'approche.

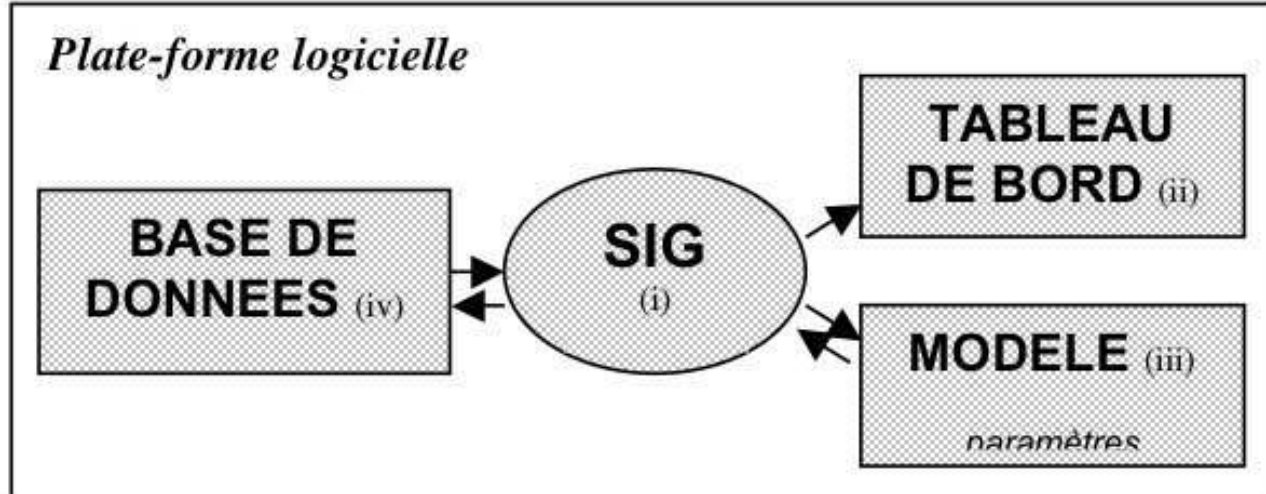

Ces quatre composants reliés doivent permettre la prise de décision à l'aide de critères et d'indicateurs (Martin et al., 2002). Les nouvelles techniques d'acquisition en continu de données de terrain sont aujourd'hui nécessaires pour suivre l'évolution de la ressource face à de nouvelles contraintes externes telles que la surexploitation ou la pollution des 
nappes. Ils permettent de prendre connaissance des impacts induits et de modéliser la réponse de l'aquifère afin de soutenir les décisions concernant, par exemple, l'arrêt d'un pompage ou la mise en place d'une barrière hydraulique (barrière de protection des forages face à une pollution ponctuelle). L'investissement de centrales d'acquisition permanentes peut être justifié dans les cas où les pratiques d'exploitation peuvent mettre en danger la pérennité de la ressource.

11 L'approche retenue est actuellement expérimentée chez notre partenaire. Les objectifs visés sont dans une première phase, de mieux connaître et suivre le phénomène de dénitrification des eaux souterraines du Béthunois en vue de le préserver. Dans une deuxième phase, d'aider les décideurs à formuler les critères qui leur permettent d'orienter les décisions à prendre.

12 La nappe de la craie sénonienne dans le secteur du Béthunois bénéficie d'un processus naturel de dénitrification bactérienne par des souches anaérobies dans la zone captive de l'aquifère, éliminant les nitrates contenus dans l'eau souterraine et conférant à la ressource des avantages importants pour fournir une eau de bonne qualité apte à la consommation. Ce phénomène a été mis en évidence au niveau régional par les recherches de Mariotti et Landreau (Mariotti et Landreau, 1988) et il a été également étudié localement par le bureau d'études SOGREAH (SOGREAH, 1998) aux alentours de Béthune. Les conclusions de ces travaux convergent sur la fragilité du phénomène. En effet, dans le cas d'une intensification de l'exploitation de la nappe sénonienne non contrôlée, l'abaissement du niveau de la nappe et l'accélération des vitesses d'écoulement peuvent entraîner une forte altération du mécanisme dénitrifiant, voire son inhibition totale, provoquant l'arrivée de nitrates aux forages d'exploitation du Béthunois (voir figure ci-dessous).

Figure 2. Représentation du mécanisme de dénitrification naturel (SOGREAH, 1998).

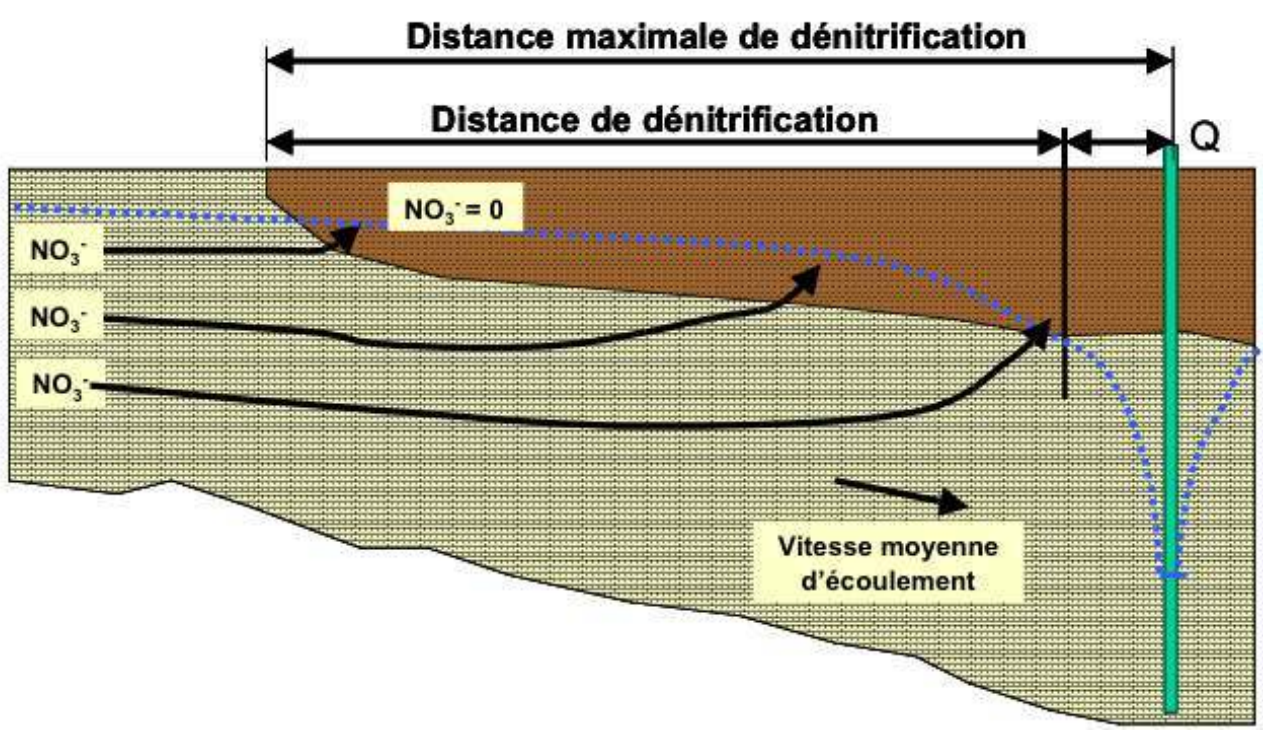

13 Les actions présentées ci-dessous ont pour objet la compréhension (a), la gestion (b) et la surveillance (c) de l'aquifère afin de préserver le phénomène de dénitrification:

14 Le phénomène de dénitrification est caractérisé, en général, par (SOGREAH, 1998):

1. les paramètres propres au phénomène: vitesse d'écoulement de la nappe, vitesse de diffusion des nitrates, vitesse de dénitrification, 
2. des indicateurs : niveaux d'eau, teneurs des eaux souterraines en $\mathrm{NO}_{3}^{-}, \mathrm{SO}_{4}{ }^{2-}, \mathrm{Fe}^{2+}, \mathrm{O}_{2}$,

3. des conditions hydrogéologiques : captivité de la nappe, milieu réducteur,

4. des conditions minéralogiques : présence d'un support riche en soufre (pyrite),

5. des conditions bactériologiques : présence de bactéries dénitrifiantes.

L'évolution des paramètres évoqués ci-dessus peut être analysée directement ou indirectement par un logiciel de simulation type $\mathrm{GMS}^{\circledR}$. Les indicateurs peuvent être déterminés par des mesures des eaux souterraines in situ ou en laboratoire (niveau d'eau, analyse chimique).

Les outils informatiques qui ont été retenus pour instrumenter la démarche de suivi du phénomène de dénitrification sont (cf. Fig.1):

1. le SIG-logiciel ArcView ${ }^{\oplus}$. Il gère l'ensemble des données physiques (géologie, ouvrages hydrauliques,...) sur le secteur d'étude. Le fond de plan numérique adopté est le $\mathrm{SCAN}_{2} 5^{\circledR}$ de l'IGN qui contient les données des cartes IGN au $25000^{\circ}$,

2. un tableau de bord qui sera mis en place à partir du logiciel ArcView ${ }^{\circledast}$ (développement spécifique à l'aide du langage auteur),

3. le logiciel de modélisation des eaux souterraines GMS ${ }^{\circledast}$ (Groundwater Modelling System) de la Brigham Young University. Le logiciel permet de modéliser le comportement hydrodynamique du système et de simuler la piézométrie et le transport de nitrates et d'autres polluants selon différents scénarios d'exploitation définis par le gestionnaire et par le décideur,

4. une base de données ACCESS intégrée dans le SIG avec des données de nature différente, physiques (forages, coupes géologiques,...) et temporelles (mesures de la qualité de l'eau, piézométrie,...).

L'objectif du système d'instrumentation est double, il permet l'acquisition des mesures relatives au phénomène (teneurs en nitrates, niveaux d'eau de la nappe, paramètres physico-chimiques,...) et le suivi en continu de ces variables permettant d'avoir une connaissance sur son état et son évolution. Dans le cas d'un abaissement trop important du niveau d'eau ou d'une augmentation significative de la concentration en nitrates, le gestionnaire sera alerté d'une possible perturbation du phénomène. Nous proposons cidessous deux scénarios d'instrumentation complémentaires. Ils consistent en :

1. scénario A: mise en place d'une centrale d'observation, instrumentée par une sonde multiparamètres permanente et immergée, localisée entre la limite de captivité de la nappe de la craie (qui coïncide à peu près avec le front de dénitrification) et l'actuel champ captant des forages du SIVOM. Son objectif est de constituer une station d'alerte en cas de perturbation importante du phénomène,

2. scénario $B$ : mise en place d'un axe de piézomètres instrumentés par des enregistreurs de niveau et éventuellement par des sondes multiparamètres, implanté dans le sens de l'écoulement des eaux souterraines et perpendiculairement à la ligne de captivité. Le but est de suivre la disparition des nitrates dans la nappe captive depuis le côté libre de la nappe de la craie et d'alerter le gestionnaire en cas de perturbation (concentrations trop élevées à une certaine distance du captage) (cf. Fig. 3). 
Figure 3. Scénario $B$ : instrumentation d'un axe de piézomètres.
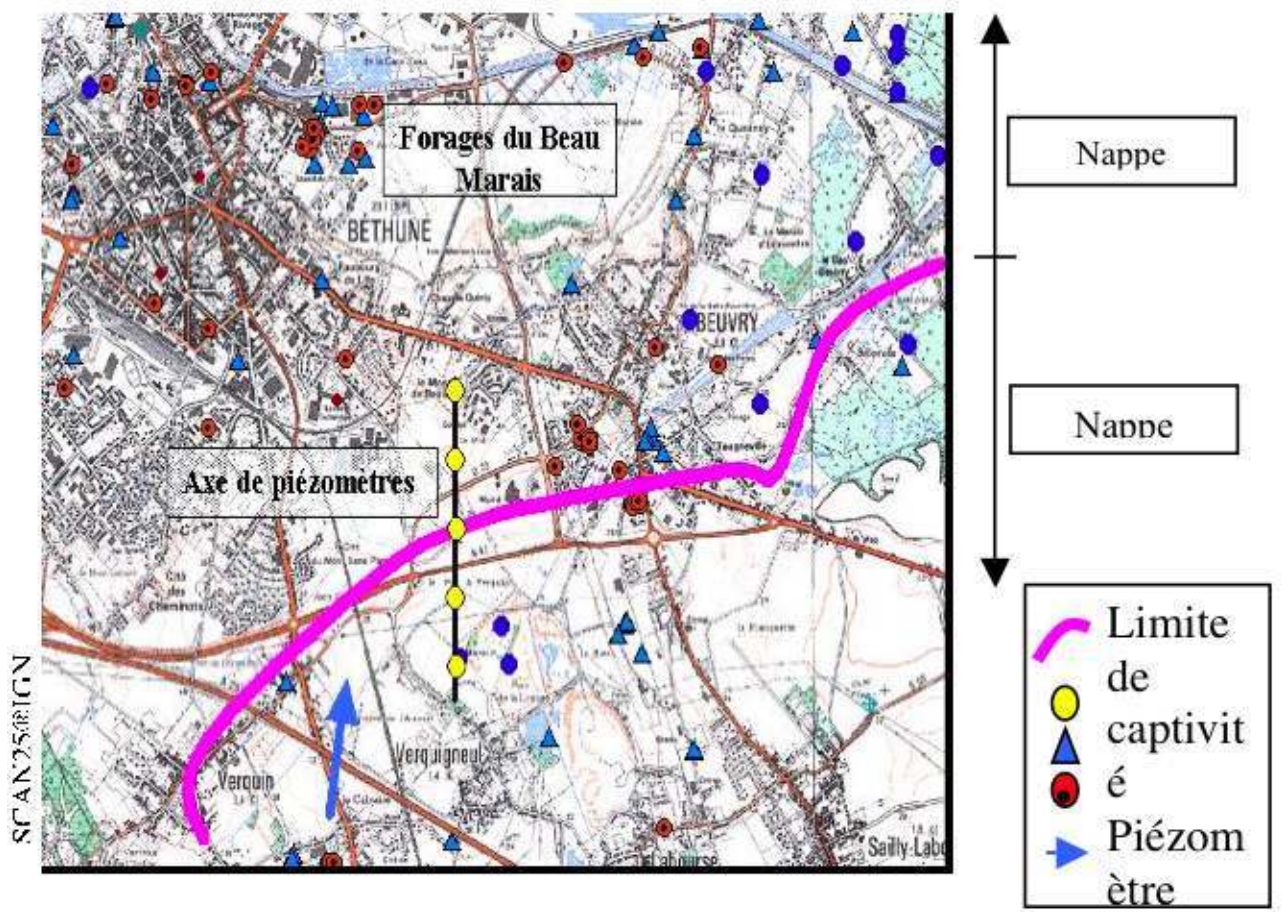
souterraines sont instrumentés par la plate-forme logicielle présentée ci-dessous (cf. Fig. 4). Nous décrivons ci-dessous les différents éléments qui composent la plate-forme et qui doivent permettre au décideur et au gestionnaire de déterminer le meilleur scénario d'exploitation de la ressource garantissant la demande en eau et préservant le phénomène de dénitrification :

1. le système d'acquisition de données et de surveillance, fournit (en temps réel ou différé) les données évolutives nécessaires à définir les indicateurs d'état de la ressource et à la modélisation ;

2. les données évolutives sont celles qui varient en fonction du temps et des conditions du système hydrologique (pluviométrie, pollution, exploitation), elles caractérisent l'état de l'aquifère: niveau de la nappe, paramètres physico-chimiques $(\mathrm{pH}, \mathrm{T}$, oxygène dissous, conductivité), concentrations $\left(\mathrm{NO}_{3}^{-}, \mathrm{SO}_{4}{ }^{2-}, \mathrm{Fe}^{2+}\right)$;

3. les données physiques ne sont pas dépendantes du temps et elles caractérisent la géométrie et le fonctionnement de l'aquifère : géologie, paramètres hydrodynamiques (perméabilité, transmissivité, coefficient d'emmagasinement). Les données physiques concernent aussi les ouvrages d'exploitation (forages) et de suivi (piézomètres) : localisation, profondeur, volume de prélèvement ;

4. le tableau de bord (interfacé avec le SIG) est composé d'une part, par les indicateurs d'état de la nappe (niveau d'eau, $\left[\mathrm{NO}_{3}^{-}\right], \mathrm{O}_{2}, \mathrm{pH}, \mathrm{T}$ ) qui en assurent sa surveillance (cf. Fig. 5), et d'autre part, par les indicateurs issus de la simulation de différents scénarios d'exploitation à partir du logiciel de modélisation (vitesse d'écoulement, vitesse de transport de nitrates, piézométrie). L'état de chaque indicateur est donné par un seuil associé défini par le gestionnaire (cf. Fig. 6); 
5. le SIG est utilisé par ses fonctionnalités de gestion de données et d'élaboration de cartes de synthèse. Il alimente d'une part le tableau de bord décrit ci-dessus, et d'une autre part le logiciel de modélisation des eaux souterraines ;

6. le logiciel de modélisation a pour but de simuler les scénarios d'exploitation envisagés par le gestionnaire et de modéliser les paramètres ayant une influence sur le phénomène de dénitrification (écoulement souterrain, piézométrie, transport de nitrates). Ces paramètres sont restitués dans le tableau de bord et constituent les indicateurs de simulation. Les données provenant de la simulation sont également restituées dans le SIG et peuvent être croisées avec les informations contenues dans le fond de plan pour éditer des cartes piézométriques, cartes de concentration, cartes à risque ;

7. les indicateurs d'état et de simulation contenus dans le tableau de bord permettront d'évaluer les différents scénarios d'exploitation à partir des critères décisionnels formalisés par le décideur et le gestionnaire. Les décisions à prendre correspondent aux modes de gestion et de contrôle du phénomène de dénitrification (exploitation et préservation) ;

8. le choix porte sur le meilleur scénario d'exploitation limitant l'impact hydraulique sur le phénomène de dénitrification et l'estimation du volume de pompage optimal garantissant les demandes en eau des populations et des industries.

Figure 4. Plate-forme logicielle appliquée à la surveillance et au contrôle du phénomène de dénitrification naturelle.

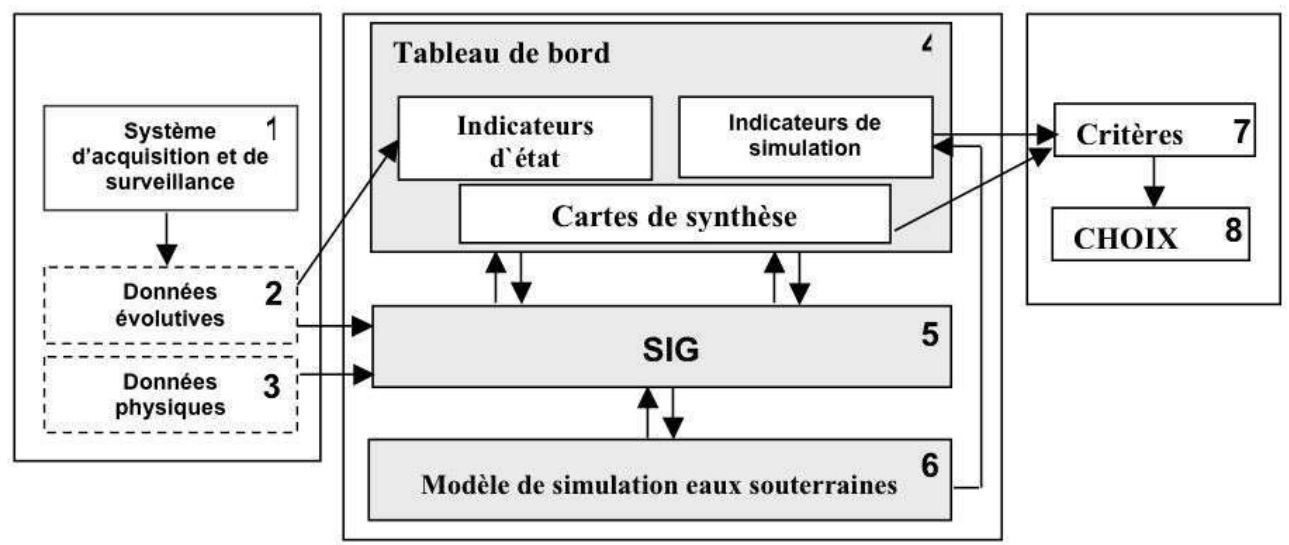


Figure 5. Exemple de tableau de bord : partie surveillance (les chiffres indiqués ne correspondent pas à la réalité et sont à titre illustratif).

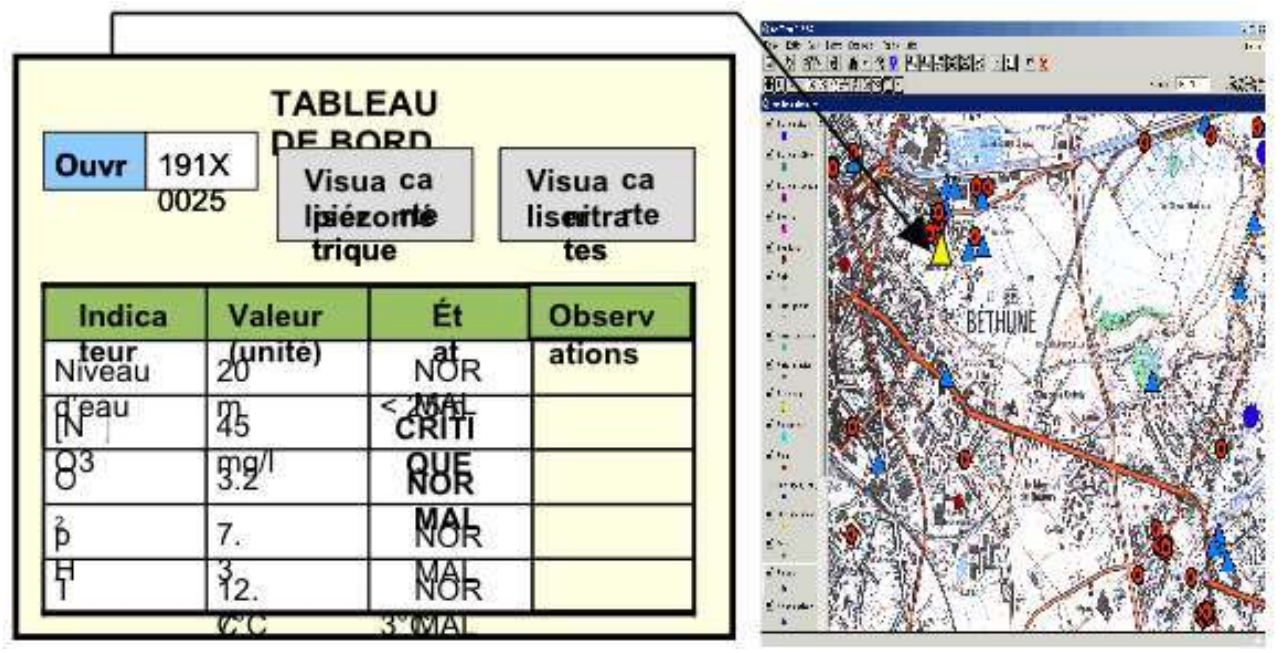

21 [Télécharger la figure ici]

Figure 6. Tableau de seuils et états associés pour quelques indicateurs.

\begin{tabular}{|c|c|c|}
\hline Indicateur & Seuil associé & Etat \\
\hline \multirow{3}{*}{$\begin{array}{l}\text { Niveau d'eau } \\
\text { (renseigne sur la } \\
\text { situation de captivité } \\
\text { de la nappe, } \\
\text { nécessaire au } \\
\text { phénomène) }\end{array}$} & $x<15 m$ & ALERTE \\
\hline & $15 m<x<25 m$ & CRITIQUE \\
\hline & $x>25 m$ & NORMAL \\
\hline \multirow{3}{*}{$\begin{array}{c}\text { NO3 }^{-} \\
\text {(d'après la norme de } \\
\text { l'UE) }\end{array}$} & {$[\mathrm{NO} 3]<25 \mathrm{mg} / \mathrm{l}$} & NORMAL \\
\hline & $25 \mathrm{mg} / \mathrm{l}<[\mathrm{NO} 3]<50 \mathrm{mg} / \mathrm{l}$ & CRITIQUE \\
\hline & {$[\mathrm{NO} 3]>50 \mathrm{mg} / \mathrm{l}$} & ALERTE \\
\hline
\end{tabular}

Nos propositions visent à répondre aux besoins et manques d'une collectivité locale dans son activité de gestion des eaux et de résolution de problèmes locaux liés à l'exploitation et à la pollution de la ressource. La mise en place d'un outil de gestion et d'aide à la décision est envisagée pour améliorer la connaissance de la ressource et pouvoir prédire son comportement. Pour cela, une plate-forme logicielle regroupant plusieurs modules de traitement de l'information est proposée. L'approche et les outils qui sont actuellement en cours d'expérimentation permettront de compléter les travaux actuels sur le sujet et en particulier sur le phénomène de dénitrification dans la zone d'étude. Dès à présent, les cadres et techniciens du service des eaux du SIVOM, très impliqués dans la recherche, disposent d'une base de données géoréférencée et d'une cartographie numérique très complètes sur le secteur du Béthunois. Elles ont été élaborées pour les besoins de l'étude. A l'issue de plusieurs présentations des travaux de recherche devant le SIVOM, les élus, désireux d'accroître la connaissance des facteurs agissant sur le phénomène et soucieux d'anticiper tout risque de perturbation excessif, ont décidée dans une première action, la mise en place d'un système d'alerte (scénario A de notre proposition). 
accompagnement de ce type d'installation, il est souhaitable, pour définir une stratégie de gestion durable de la ressource, d'élaborer un ensemble de critères décisionnels généraux et à spécificité locale. Ces critères seront évalués à partir des indicateurs du tableau de bord présenté ici, interfacé à un SIG et au système d'acquisition de données. Le travail d'élaboration de critères décisionnels pourra être réalisé en concertation avec les chercheurs, le gestionnaire exploitant et les élus locaux.

\section{BIBLIOGRAPHIE}

Boulémia C., Henry E., Top G., Martin J.L. et Masson F.X., 2000, « Axes de développement d'outils d'aide à la décision dans les moyennes collectivités à partir d'un Système d'Information Géographique. Cas des réseaux d'assainissement et d'alimentation en eau », in: Actes de la $2^{\text {ème }}$ Conférence Internationale sur l'Aide à la Décision dans le domaine du Génie Civil et Urbain, Lyon, 20-22 novembre 2000, Vol. 1, pp. 473-482.

Crausaz P.A. et Musy A., 2001, « Apports des SIRS dans la gestion des eaux de surface: le projet GESREAU », Hermès, pp. 111-129.

Ganoulis J., 2001, « La gestion de l'eau à l'aube du $3^{\mathrm{e}}$ millénaire : vers un paradigme scientifique nouveau ", Revue des Sciences de l'Eau, 14/2, pp. 213-221.

Mariotti A. et Landreau A., 1988, " ${ }^{15} \mathrm{~N}$ isotope biogeochemistry and natural denitrification process in groundwater: Application to the chalk aquifer of northern France », Geochimica et Cosmochimica Acta, Vol. 52, pp. 1869-1878.

Martin J.L., Carlier E., Boulémia C. et Henry E., 2002, « Approach to an integrated groundwater resource management in a medium-sized local community: setting up of a management and decision-making support tool. Case of the chalk aquifer in the Béthunois area (Nord-Pas de Calais), France ", in: Proceedings of the Fifth International Conference on Hydroinformatics, Cardiff, 1-5 juillet 2002, Vol. 2, pp. 1000-1005.

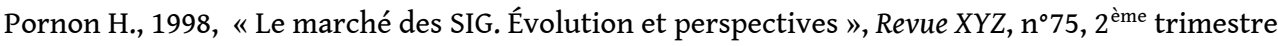
1998, pp. 68-70.

SOGREAH [Syndicat mixte pour le Schéma Directeur de l'Arrondissement de Béthune], 1998,. Étude du phénomène de dénitrification naturelle dans la craie sénonienne à l'ouest de Béthune, 1998.

Top G., Boulémia C. et Henry E., 2000, «Etude de faisabilité d'une base de données urbaines », Rencontre du Réseau Doctoral de Génie Civil, Aussois, 30 janvier-2 février 2000.

Villeneuve, J.P., Fortin, J.P. et Mamouny, K., 1995, « Gestion intégrée de la ressource en eau à l'échelle du bassin versant à l'aide d'un système informatisé (GIBSI) », Américana 95, Salon de l'environnement des Amériques, (Montréal, Canada, mars 1995). Non publié.

Waaub, J.P., 2001 « Expériences en gestion par bassin versant, GEIGER », Séminaire de Gouvernance Territoriale et outils d'aide à la négociation, 19-21 mars 2001, Ecole des Mines de Saint-Étienne, pp. 93-96. 


\section{NOTES}

1. Remerciements : Nous tenons à remercier le service des eaux du SIVOM de la Communauté du Béthunois pour son soutien et sa disponibilité. Il a également permis le financement et la conduite de ces travaux de recherche.

\section{RÉSUMÉS}

Les villes sont sujettes à des augmentations de population et d'activité industrielle, ce qui se traduit par une demande supplémentaire en eau. Notre objectif est de développer un outil de gestion et d'aide à la décision pour une meilleure connaissance de la ressource et pour soutenir les décisions de planification dans les services des eaux des moyennes collectivités locales. L'outil permettra une exploitation contrôlée du système aquifère et en particulier la préservation du phénomène de dénitrification naturelle ayant lieu dans la nappe de la craie du Béthunois au Nord de la France. Dans cet article, nous proposons la mise en place d'un tableau de bord à partir d'une plate-forme SIG couplée à un outil de modélisation hydrologique. Nos travaux de recherche sont appliqués au SIVOM de la Communauté du Béthunois.

Cities face a substantial increase in population and in industrial activities which entails an increasing demand for water. Our aim is to develop a management and decision-making framework for a better knowledge of the resource and to support the planning and decisions in the water boards of medium-size local communities. The framework will allow the reasoned exploitation of the aquifer system and in particular the preservation of the natural denitrification process which occurs in the chalk aquifer in the Béthunois area in northern France. In this article, we propose the setting-up of a control panel thanks to a GIS platform coupled with a hydrological model. Our research work is applied to the SIVOM of the Béthunois.

\section{INDEX}

Mots-clés : ressource eau souterraine, gestion, aide à la décision, dénitrification naturelle Keywords : groundwater resource, management, decision-making support, natural denitrification

\section{AUTEURS}

\section{JOSÉ LUIS MARTIN-BORDES}

Docteur en Génie Urbain de l'Université de Marne-La-Vallée, spécialisé dans le domaine de la gestion des eaux souterraines. Il a été chercheur dans l'Équipe Hydrologie, Sol et Environnement (HSE) de l'Université d'Artois. Il a obtenu le 3eme Prix Jeunes Chercheurs René Houpert lors des XXI Rencontres Universitaires du Génie Civil en 2003 avec sa 
communication « Outils de gestion et d'aide à la décision dans une moyenne collectivité locale: Application à l'exploitation de la ressource en eau souterraine ». Il est consultant en hydrogéologie et en gestion de l'eau en milieu urbain au sein du Programme Hydrologique International (PHI) de l'UNESCO. Contact : hydrojose@voila.fr; jl.martin@unesco.org

\section{ERIC HENRY}

Faculté des Sciences Appliquées, Université d'Artois

\section{CHERIF BOULEMIA}

Ingénieur, Docteur en Génie Civil

\section{FRANÇOIS-XAVIER MASSON}

Faculté des Sciences Appliquées, Université d'Artois / DRAF Nord-Pas de Calais 